\title{
Developing Actuation Mechanism for Stick-Slip Based Intelligent Mobile Displays
}

\author{
Farooq A*, Weitz P*, Evreinov G*, Raisamo R*, Takahata D+ \\ Faculty of Communication Sciences, Tampere University, Tampere, Finland \\ Email:*firstname.lastname@staff.uta.fi,+d_takahata@fukoku-rubber.co.jp
}

\begin{abstract}
In line with our previous work, this research focuses on enhancing touchscreen based interaction through stick-slip phenomena. By balancing inertial and frictional forces on a transparent screen overlay, we can control the resulting directional forces specific to multiple objects on a touchscreen surface. Using "stick-slip" phenomenon, we can associate tangible objects in relation to their virtual environment and adjust their behavior in real time without any stiff mechanical linkages. Our previous research shows the possible advantages of such a system (Stick-Slip Kinesthetic Display Surface) for a wide range of applications, such as continuous supervised input as well as novel applications with cross-environment interaction, where real-world physical objects can interact with their virtual counterparts and vice versa. However, to ensure that the directional forces are sufficient for these and other types of applications to serve as the system output, the mechanical actuation mechanism needs to be specifically designed for the particular novel use case. This research utilizes an electromagnetic setup to develop custom designed linear actuator which can increase the efficiency of the stick-slip based system. Our testing shows that the custom actuator is stable and more efficient at generating directional forces in the smart kinesthetic display surfaces (SKDS) as compared to actuators designed for conventional vibrotactile feedback.
\end{abstract}

Keywords-component; haptics feedback; kinesthetic touchscreens; tactile feedback; screen overlays; user interaction.

\section{INTRODUCTION}

Kinesthetic information is the main component of haptic feedback that actually integrates diverse afferent signals into the entire percept. By generating a combination of directional forces (or torques), it is possible to simulate some physical properties of objects during interaction with them in virtual environments. Different systems and transducers can be used to deliver forces [1] in augmented haptic space. Based on these transducers, a number of devices have been developed which use kinetic energy to support the kinesthetic sense and to convey information regarding different qualities and properties of virtual objects. However, most of these devices use linkages and stiff chains to generate the strong directional forces needed to simulate the properties of virtual objects. Large standalone devices like the Geomagic's Phantom and Novint's Falcon may provide meaningful interactions in a controlled haptic space, but they are unsuitable for common touchscreen interaction in mobile and handheld devices.

Therefore, this research was aimed at designing a mechanism by which it is possible to apply linkage-free directional forces to a stylus tip to generate tactile-kinesthetic afferentation with the help of a screen overlay. Utilizing the stick-slip phenomenon [2] as the core technology to deliver directional forces in the absence of stiff kinematic chains and mechanical linkages, this research illustrates how tangible objects (e.g. a stylus) on the touchscreen can actively be manipulated in a fully controlled and efficient manner. Essentially, the technology described in this research opens up new possibilities for the computer to generate an active realtime response (output) to the user's inputs, creating a truly natural interaction paradigm. The developed system can be used to provide continuously supervised learning and deliver feedforward information that may be able to predict and modify user behavior.

\section{RELATED WORK}

There are two key problems with user interaction in current computer systems. Firstly, they still fundamentally rely on the "command-language execution" model, and secondly, they are only able to output information in a variety of audio and visual formats. Essentially, current computers are totally deprived of the opportunity to exchange kinetic energy with external objects. Can it be truly possible for a computer peripheral (interactive surface, sensor pad, touchscreen) to be able to move or push the user's hand, throw a tangible object, or physically respond to the user's touch-based interaction? We believe this is feasible, and our research aims to make it practical as well. The techniques and technologies of interactive space that allow us to produce directional forces (either normal or tangential) against tangible objects are under continuous development. Generating lateral forces on top of a touchscreen using a screen overlay has been addressed earlier $[3,4,5,6$, and 7$]$.

Until now, the only way of creating a strong sense of directional forces on an interactive surface - in the absence of the mechanical linkages with respect to a reference ground plane-was by modulating the friction coefficient [10]. When the friction is artificially raised, moving an input device, such as a stylus or a fingertip, over the interactive surface requires a greater force from the user to move the manipulandum. On the other hand, if the friction coefficient is artificially lowered, moving the manipulandum over the interactive surface becomes much easier. By switching between both states, a perception of virtual surface structures (surface edges, virtual fixtures, and textures) can be controllable with respect to the interaction scenario $[11,12]$. Friction modulation can be achieved in multiple ways. One approach was described by Müller et al. [10]. The researchers developed a stylus containing a steel ball tip and an electromagnetic coil. When moving the stylus over the display surface, the steel ball tip rotates with a minimum amount of friction. By using an electromagnetic brake, the friction force can be modified 
dramatically. Thus, the force required to move the stylus over the display surface can be modulated.

Another approach was presented by Levesque et al. [9]. Using $26 \mathrm{kHz}$ vibrations produced by a piezoelectric actuator, the authors were able to create a squeezed air film that reduces the friction on an interactive display surface. By using this technique, a just noticeable difference in friction of approximately $30-40 \%$ has been recorded. The presented approach allowed for the simulation of multiple friction levels that could be used for the tactile presentation of different object properties and well-distinguishable levels of the virtual surface. An alternative approach could allow creating lateral forces in order to simulate virtual edges and surface structures. This concept is based on research done by Robles-De-La-Torre [13], who described the importance of lateral forces in perceiving, recognizing, and identifying planar shapes. Based on his research, the T-Pad (Tactile Pattern Display through Variable Friction Reduction) was developed by Winfield et al. and presented at EuroHaptics [8]. The authors utilized piezoelectric actuators to alter the friction coefficient and simulate lateral forces under the fingertip. On the other hand, Kaye [14] also utilized the slightly different approach of designing an active computer response (output) to the user's touchscreen inputs. He introduced the possibilities of using a saw-tooth-shaped vibrational pattern to create a well-perceived force that could be applied to the user's fingertip. This allowed the generation of a richer set of sensations when interacting through the mobile touchscreen.

Although the concept discussed by Kaye [14] is useful in providing tactile signals to the skin, none of the approaches discussed above produces forces strong enough to change the direction of stylus or fingertip movements. The differences in friction force could only be experienced by the user and perceived when s/he initiated the actual movement of the input device (manipulandum). Essentially, most of the solutions discussed can be described as "passive" because they only react to the user's actions, instead of actively affecting the user's behavior. Our research on the other hand, utilizes screen overlays to generate the friction coefficient for creating directional forces. Expanding on the research of Kaye [14] and Reznik et al. [2], our previous work [15, 16, 17] utilizes saw tooth vibration signals to create onscreen directional forces through stick-slip motion.

\section{SYSTEM DESIGN}

In our previous research, while looking into the various possibilities of creating directional forces, and the immense usefulness of having kinesthetic feedback on touchscreen devices, we developed the "Stick-Slip Kinesthetic Display Surface" (SKDS) [16]. The SKDS utilized an overlay mechanism to generate the tangential displacement necessary to create directional force on the touchscreen of a Microsoft Surface Pro 3 tablet (Fig. 1 and 2). Actuators on all four sides of the overlay were affixed to a Plexiglas plate and were controlled independently to create the necessary actuation in their relative directions. This created a fully-controllable space on top of the touchscreen in which it was possible to create stick-slip motion for any tangible object with a maximum weight of about $250 \mathrm{~g}$. Using the saw tooth signal waves specified by Kayes [14], we were able to move tangible objects over the touchscreen surface without any mechanical linkages. Using this setup we have developed a number of stylus based applications $[15,17]$ from handwriting learning to 2D gaming interaction on a simple touchscreen device.

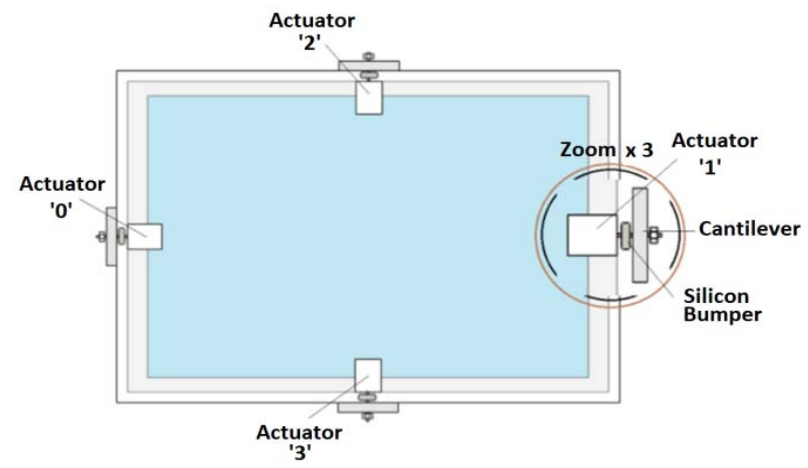

Figure 1. Top and cross sectional view of the Design for the "Stick-slip Kinesthetic Display Surface".

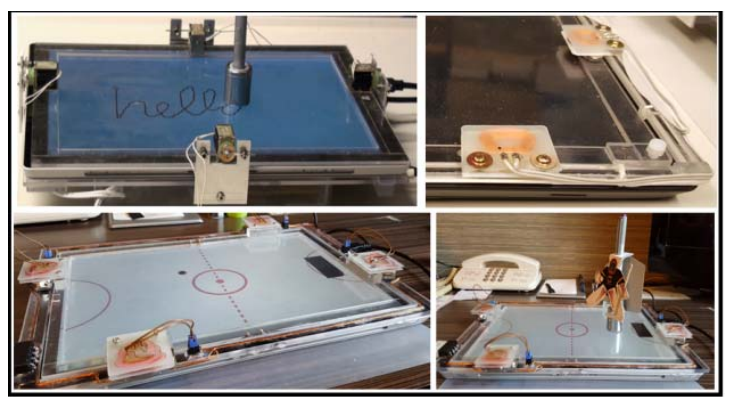

Figure 2. Various embodiments of the "Stick-slip Kinesthetic Display Surface" prototype.

However, if we look at the actuation mechanisms employed in the SKDS system, we see that although conventional vibrotactile feedback actuators can be used in the SKDS, they are perhaps not the most efficient method in creating the stick-slip motion necessary to move tangible object of the screen. In fact, current mobile haptic components in general, such as piezoelectric and electromagnetic actuators, are developed to provide tactile feedback in mobile devices and are designed to create sustained displacement in loaded environment. Furthermore, most of these components were designed to operate at specific frequencies and generate a range of actuation signals (not specifically saw tooth wave) for direct or indirect haptic feedback. Furthermore, these actuators, with their limiting physical parameters are not ideal for use in novel systems, which generate active physical outputs through smart kinesthetic display surfaces (i.e. SKDS).

On the contrary smart systems, such as SKDS, require calibrated actuation parameters, such as force, displacement, accuracy and efficiency along with the consistency to be able to reproduce the physical parameters needed, during every actuation cycle. Our previous research $[16,17]$ showed that ideal actuators for SKDS systems need to ensure displacement values of about $1.5 \mathrm{~mm}$ or greater, under an average load of about $200 \mathrm{~g}$, within a consistent period of $3 \mathrm{~ms}$. Farooq et. al. 
(2016), also demonstrated that power efficiency, although important for basic actuation, is not a determining factor in affecting the overall performance, beyond the initial threshold of actuation. Therefore, it is possible to custom design a linear electromagnetic actuator (using EM field simulators such as MagNET for SolidWorks [19] and QuickField [20]), as done by Chou et. al.,[18]. Designing a custom actuator provides the opportunity of ensures that the focus of the actuator would be on fulfilling the essential requirements without unnecessarily emphasizing on its efficiency (power consumption vs. force output), but rather concentrating on the transfer of mechanical energy to the tangible object sliding over the touchscreen through the reciprocating motion of the intermediate transparent overlay. Essentially, this would mean that the actuation component should work effectively with the screen overlay to yield and apply the needed directional forces to the object on-top of the touchscreen surface (Fig. 3).

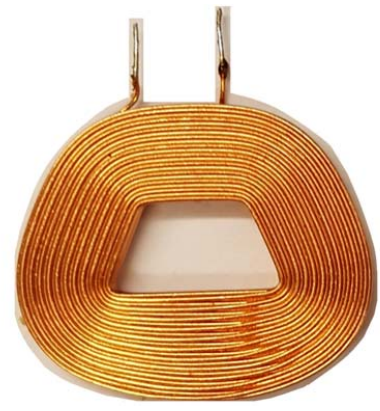

(a)

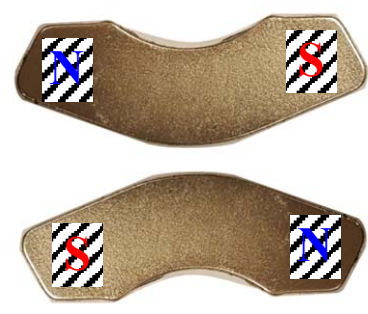

(b)

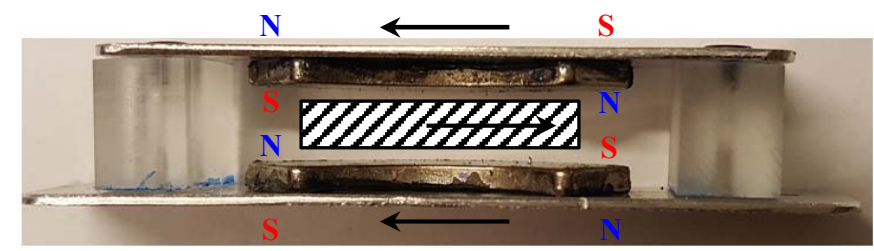

(c)

Figure 3. Design and arrangement of the cutom E2VA.

To achieve this, we developed the E2VA actuator (Enhanced Electromagnetic Voice-coil Actuator) (Fig. 3), a specifically designed actuation source for the SKDS system. Utilizing a precise configuration of the steel poled super strong neodymium magnet and low impedance $(2.5 \mathrm{oHms})$ high current $(2 \mathrm{~A})$ voice coil, the custom E2VA actuator was developed to generate the necessary actuation requirement (collected from previous research) and perform better in smart systems such as the SKDS. The actuator consisted of a custom wound coil fixed on a ridged Plexiglas frame. The coil and accompanying frame was affixed to one side of the screen overlay, while from the other side it is placed in between two thin horizontally mounted magnets. The polar arrangement of the magnets was such that the north and south poles were on either side of the magnet (Fig 3b).

These magnets were glued to a magnetically soft iron frame and joined together using two pieces of $1.5 \mathrm{~cm}$ thick Plexiglas, ensuring that the magnetic poles the two magnets faced each other (Fig. 3c). Once the coil was turned on it was attracted / repelled by the corresponding polls of magnets creating actuation of the screen overlay. The design of the actuator was such that the two magnets, the winding of the coil and the resulting magnetic field was precisely calibrated to achieve maximum yet stable displacement over multiple actuation cycles. Similarly it was also ensured that the E2VA provided relatively similar amounts of force through-out the actuation cycle, irrespective of the actuation signal. Finally, calibration process of the actuator ensured that the custom E2VA remained stable within the needed frequency range required for stick-slip motion $(30-100 \mathrm{~Hz})$.

\section{System EVALUATION}

To compare the efficiency and performance of the E2VA actuator in the SKDS system, we utilized a similar setup as defined in Farooq et. al., 2016 . The objective was to generate directional forces through the screen overlay onto the Microsoft Surface Stylus pen (and its base, weighing a total of $168 \mathrm{~g})$ and to move the stylus from point A to point B $(254 \mathrm{~mm}$ away). Along with the E2VA (Fig. 4b), we utilized three other actuators, a piezoelectric APA120s (Fig. 4c) actuator (manufactured by Cedrat), an ELV0632B (Fig. 4d) movable mass (inertial) actuators (developed by AAC Technologies) and a TEAX14C02-8 (Fig. 4a) voice coil exciter (developed by Tectonic Elements).

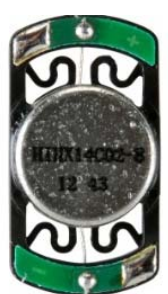

(a)

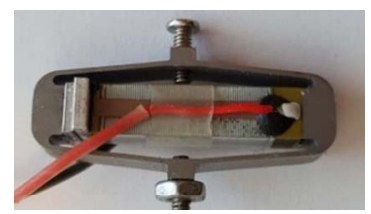

(c)

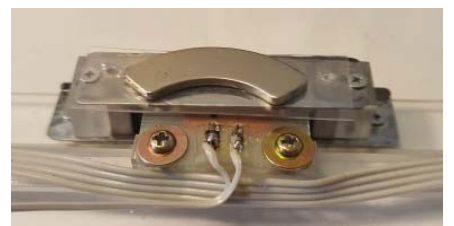

(b)

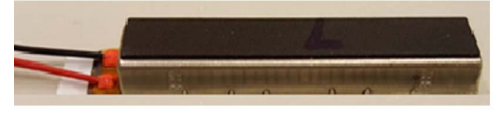

(d)
Figure 4. The four actuators used in the study (Tectonic TEAX14C02-8 (a), the custom E2VA (b), Cedrat APA120s (c) and AAC ELV0632B (d).

All four actuators were attached similarly to the SKDS as to limit environmental variations during the experiment. Keeping the frequency of actuation to about $30-40 \mathrm{~Hz}$ for each actuator, we measured the horizontal displacement of the screen overlay, the forces generated by the stylus (and its base), the electrical power required to create these forces, as well as the speed of the stylus while moving on the touchscreen from point A to point B. The horizontal micromovements were recorded using a MicroSense displacement sensor (5810, with a 5622-LR, $20 \mathrm{kHz}$ probe), while force generated by the stylus was measured using a Tensile Stress Gauge ZEF and the speed was calculated using a custom software on the MS Surface tablet which tracked the pixel position of the stylus tip, while moving from point $A$ to $B$ on the touchscreen. 


\section{RESUlTS}

The results from the technical evaluation validated finding published in Farooq et al., 2016 [17]. Ideal actuation needed to generate stick-slip motion on the SKDS system must have a minimum displacement of $1.5 \mathrm{~mm}$ (in a loaded environement), and be able to generate a minumum force of about $5 \mathrm{~N}$. This force needs to be created by the actuator and transferred through the displacement of the screen overlay to the physical object on the overlay (stylus). To gauge whether the actuator is efficient for this task, we need to record the ratio of force generated for overlay displacement versus the force by which the object on the screen moves over the MS Surface Pro 3 tablet.

Although all four actuators were able to achieve some form of displacement of the screen overlay, to achieve suitable directional forces on the display and create stick-slip motion of the stylus, some were more efficient than others. With reference to displacement in non-loaded SKDS environments, E2VA and Tectonic TEAX14C02-8 voice coil actuators (VCA) remained stable during the actuation process, and were the highest performing actuators. The E2VA performed slightly better under load and was very efficient at translating linear actuation into stick-slip forces. However, the difference between the E2VA and the tectonic actuator was very small. With reference to unloaded displacement, E2VA outperformed the remaining three actuators with a maximum stable displacement of $1.39 \mathrm{~mm}$ over the $1.25 \mathrm{~mm}$ of the Tectonic (Fig. 5). Essentially, this also meant that the solenoid applied signal and the recorded actuation of the screen overlay (using the finger probe) showed less degradation.

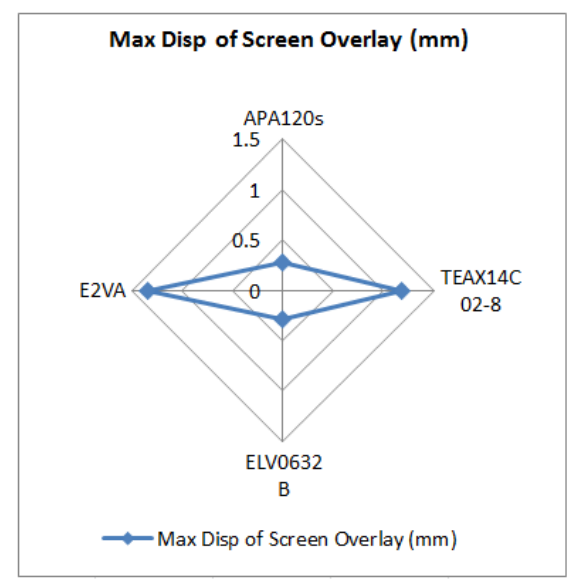

Figure 5. Variation between maximum screen overlay displacements in loaded conditions, for all four actuators.

Conversely, the Cedrat APA120s and AAC ELV0632B were unable to achieve similar efficiency. Amongst the four actuators, APA120s required the largest amount of power, and yet translated into the smallest displacement to the stylus. For this reason, although the APA120s was able to generate stickslip motion, the speed and efficiency at which this output was achieved was very low (Fig. 6). Considering both the APA120s and ELV0632B are pizoelectric actuators with inherrently limited actuation potential, they may not be the optimal actuation technology (as is currently) to be incorporated in an intelligent actuation surfaces, such as
SKDS. On the contrary, both the Tectonic and custom E2VA actuator performed better, which may mean that perhaps electromagnetic actuation mechanism could be the way forward in developing an actuation mechanisim for SKDS systems.

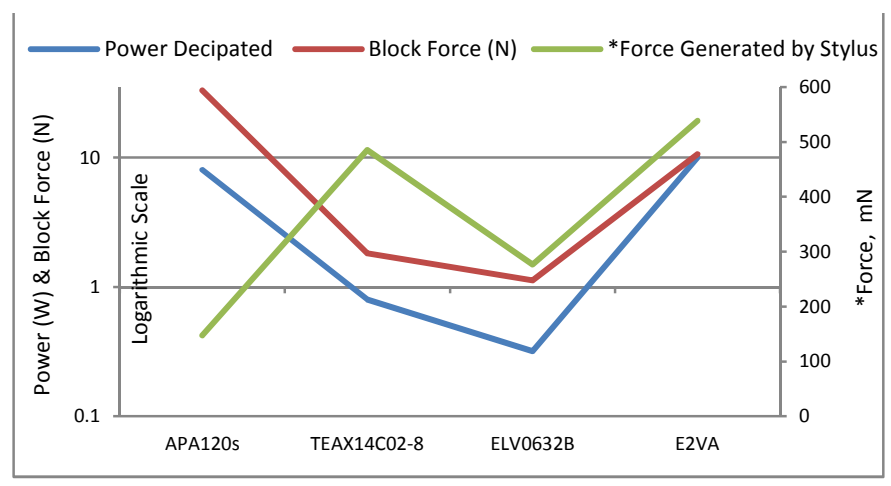

Figure 6. Variation between Power consuption and Force translated to the stylus for all four actuators

Similarly, if we look at the speeds achieved through the four actuators we can see that the stylus speeds achieved (Fig. 7) using both the Cedrat and AAC was also considerably less while the forces generated were below the Tectonic and E2VA actuators. The voice coil exciter on the other hand, created sufficient micro-displacements and the necessary linear forces (Fig. 6). However the displacement was not consistent throughout every actuation cycle to create enough difference in friction and inertial forces during the 'sticking' and 'slipping' phases. This caused the stylus to rotate in a circle while moving from point $\mathrm{A}$ to $\mathrm{B}$, essentially limiting its efficiency and speed as well as causing the application drivers to continuously adjust the motion of the stylus pen (Fig. 7).

Having said this, the voice coil generated the maximum displacement (for the screen overlay and the stylus) per milliwatts $(\mathrm{mW})$ as compared to the other three actuators; however, this motion was uncontrolled and reduced the overall speed of the stylus. On the other hand, the E2VA was not as efficient at converting the electrical energy into stylus displacements; however, it was much more controlled for the displacement it provided. Similarly, it generated enough forces to displace the screen overlay (which was greater than $108 \mathrm{~g}$ of load from the stylus and its base of $56 \mathrm{~g}$ ) consistently within the necessary timeframe to generate the maximum speed for the stylus, moving from point A to point B.

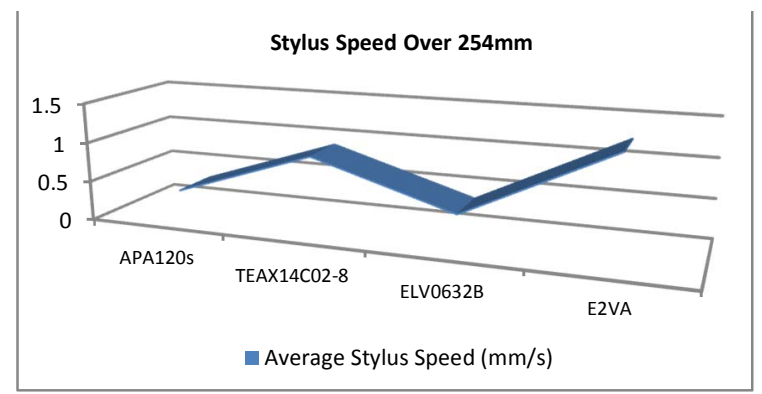

Figure 7. The mean speed of the stylus traveling from point A to B, averaged in opposite and diagonal directions, over the touchscreen for each actuator. 


\section{DISCUSSION \& FUTURE RESEARCH}

This study was designed to evaluate the possible efficiency of the custom designed E2VA actuator. Building on our previous research, we have developed an enhanced electromagnetic voice-coil actuator and compared it to the generic Tectonic VA, and other off-the-shelf available piezoelectric vibrotactile actuators. The E2VA was calibrated to perform efficiently at specific frequencies (used for creating stick-slip on the SKDS system). The E2VA was also developed to be more stable throughout the actuation cycle and produce consistent forces and displacement values. The results showed that the custom designed E2VA did remain more stable and consistent in the generating SKDS as compared to the AAC, Cedrat and even Tectonic VCA. Infact, due to its stability it out performed the other actuators in all the three tests (displacement, speed and efficiency). As seen from the results (Fig. 5 - 7) the Cedrat and AAC actuators did not produce sufficient displacements of the screen overlay and limited the transfer of directional forces to the MS Surface Stylus per actuation cycle. Due to this we saw that the speed of stylus achieved using both the Cedrat and AAC was also considerably less and the efficiency was limited as well. Though the Tectonic VCA were able to produce sufficient micro-displacements and the necessary linear forces (Fig. 5), the displacement was not consistent throughout every actuation cycle to create enough difference in friction and inertial forces during the 'sticking' and 'slipping' phases. As discussed, due to this reason efficiency of the VCA remained lower than E2VA.

Although the results are very clear with respect to actuation technology, we must also consider the mediation of the actuation through the screen overlay. In fact, the SKDS can be broken down into three structural components: the Actuation Mechanisim (Actuators), the Actuation Controller (driving software algorithm), and the mediation mechanisim utilized to create directional forces on the toucschreen (the screen overlay). In this study we developed and tested a custom designed and optimized actuation source (the first component of the SKDS). However, more research is needed to improve the driving software algorithm and the mediation mechanisim (screen overlay). Therefore the following research will focus more on these two aspects of the SKDS and evaluate and compare the efficiency of the system, achieved through optimizing all components of the SKDS system.

\section{REFERENCES}

[1] Evreinova, T. V., Evreinov, G., and Raisamo, R. From Kinesthetic Sense to New Interaction Concepts: Feasibility and Constraints, International Journal of Advanced Computer Technology, vol. 3, no. 4, 2014, pp. 133.

[2] Reznik, D., and Canny, J. A flat rigid plate is a universal planar manipulator, in IEEE International Conference on Robotics and Automation. Proceedings, vol. 2, May 1998, pp. 1471-1477.

[3] Perlin, K., Rosenfeld D.A., Kollin S.J. Manipulation of objects. US Patent 8,725,292B2, May, 2014.
[4] Reznik, D. S., The Universal Planar Manipulator. Dissertation, University of California, Berkeley, 2000.

[5] Roudaut, A., Raus, A., Sterz, C., Plauth, M., Lopes, P., and Baudisch, P. Gesture Output: Eyes-Free Output Using a Force Feedback Touch Surface. CHI'13, ACM, 2013 pp. 2547-2556.

[6] Saga, S, Raskar, R. Simultaneous geometry and texture display based on lateral force for touchscreen. IEEE World Haptics Conf., Korea, 2013 pp. 437-442.

[7] Saga, S., Deguchi, K. Lateral-Force-Based 2.5-Dimensional Tactile Display for Touch Screen. IEEE Haptics Symposium, Vancouver, BC, Canada, 2012 pp. 15-22.

[8] Wineld, L., Glassmire, J., Colgate, J., and Peshkin, M. T-PaD: Tactile Pattern Display through Variable Friction Reduction," in EuroHaptics Conference, and Symposium on Haptic Interfaces for Virtual Environment and Teleoperator Systems. World Haptics 2007, pp. 421426.

[9] Levesque, V., Oram, L., MacLean, K., Cockburn, A., Marchuk, N. D., Johnson, D., Colgate, J. E., and Peshkin, M. A. Enhancing Physicality in Touch Interaction with Programmable Friction, in Proceedings of the SIGCHI Conference on Human Factors in Computing Systems, CHI '11. ACM, 2011, pp. 2481-2490.

[10] Muller, A., Hemmert, F., Wintergerst, G., and Jagodzinski, R., Reective Haptics: Resistive Force Feedback for Musical Performances with Stylus - Controlled Instruments, in Proceedings of the International Conference on New Interfaces for Musical Expression, Sydney, Australia, Jun. 2010, pp. 477-478.

[11] Daud, O. A. Haptic Systems for Post-Stroke Rehabilitation: from Virtual Reality to Remote Rehabilitation, PhD, University of Trento, Mar. 2011.

[12] Abbott J., Marayong P., and Okamura A. Haptic Virtual Fixtures for Robot-Assisted Manipulation. Department of Mechanical Engineering, The Johns Hopkins University. Springer, Robotics Research, STAR 28, 2007, pp. 49-64.

[13] Robles-De-La-Torre, G. Comparing the role of lateral force during active and passive touch: Lateral force and its correlates are inherently ambiguous cues for shape perception under passive touch conditions, in Proceedings of Eurohaptics, 2002, pp. 159-164.

[14] Kaye, J. J., Sawtooth Planar Waves for Haptic Feedback, in Adjunct Proceedings of the 25th Annual ACM Symposium on User Interface Software and Technology, ser. UIST Adjunct Proceedings, ACM, 2012, pp. 5-6.

[15] Farooq, A., Weitz P., Evreinov, G., Raisamo, R., Takahata, D. Touchscreen Overlay Augmented with the Stick-Slip Phenomenon to Generate Kinetic Energy. In Proceeding s of ACM UIST'16 Adjunct, October 16-19, 2016, Tokyo, Japan, http://dx.doi.org/10.1145/2984751.2984758.

[16] Farooq, A., Weitz P., Evreinov, G., Raisamo, R., Takahata, D. Developing a Stick-Slip Based Kinesthetic Touchscreen System for realtime Stylus Manipulation. In Proceedings of IEEE SENSORS 2016, October 30 - Nov 2, Florida, USA.

[17] Farooq, A., Weitz P., Evreinov, G., Raisamo, R. Mechanism for Developing a Kinesthetic Haptic Feedback System. In Proceedings of IEEE ICST 2016, Nov 11-13, Nanjing, China, 2016.

[18] Chou P., Lin Y, Cheng S. A Novel Seesaw Swivel Actuator Design and Fabrication. In Proceeding of IEEE Transaction on Magnetics, VOL. 46, NO. 7, July 2010.

[19] MagNet for SOLIDWORKS, A 3D electromagnetic field simulation software. www.infolytica.com/en/products/mfs/ (accessed on 10.04.2017).

[20] QuickField 6.3 User's Guide developed by Tera Analysis Ltd. http://www.quickfield.com/demo/manual.pdf (accessed on 10.04.2017). 